Open Access

\title{
Cluster policy: insights from the German leading edge cluster competition
}

\author{
M. Rothgang ${ }^{1 *} \mathbb{D}$, U. Cantner ${ }^{4}$ D , J. Dehio ${ }^{1}$, D. Engel ${ }^{1,5}$, M. Fertig $^{2}$, H. Graf ${ }^{4} \mathbb{D}$, S. Hinzmann ${ }^{4}$, E. Linshalm ${ }^{3}$, M. Ploder ${ }^{3}$, \\ A. -M. Scholz ${ }^{2}$ and S. Töpfer ${ }^{4}$
}

* Correspondence:

Michael.Rothgang@rwi-essen.de ${ }^{1}$ RWI Leibniz Institute for Economic Research, Hohenzollernstraße 1/3, 45128 Essen, Germany Full list of author information is available at the end of the article

\begin{abstract}
With its Leading-Edge Cluster Competition (in German: Spitzencluster-Wettbewerb; LECC), the Federal Ministry of Education and Research (Bundesministerium für Bildung und Forschung, BMBF) is supporting innovation clusters in a nationwide contest for the first time. In three waves, 15 cluster initiatives were selected and provided with funds to support them on their way to becoming international leaders in their field of technology. This paper presents the results of empirical studies regarding several important aspects of the LECC. The analysis of network relations shows that the LECC's short-term goal of intensifying or enhancing networking between innovative stakeholders in the cluster regions has been achieved. At the same time, certain types of regional impulses of the LECC can already be observed, primarily an improved visibility of the Leading-Edge Cluster regions and enhanced regional R\&D activity. When looking at causal programme effects, we find that the programme increases firm-level R\&D expenditure, but also that the programme design influences the programme impulse, e.g. by promoting additional activities of SMEs.
\end{abstract}

\section{Introduction}

Cluster policies can be seen as a recent stage of innovation policy in Germany which started in the 1990s (Fier and Harhoff 2002). Such policies are motivated and inspired by a literature which builds on the influential works of Allen (1983) on collective invention, Lundvall (e.g. 1988) on user-producer interaction and collective learning, Freeman (1991) on innovation networks, and Porter (e.g. 1998) on the benefits of co-location in industrial clusters. In the following, a lively discussion about the justification, means of implementation, benefits, and timing of cluster policies developed (e.g. Martin and Sunley 2003, Brenner and Schlump 2011). Among the main policy rationales are system failures, where potentials for positive externalities or technological spillovers are not fully exploited (Cantner and Graf 2003, Moodysson and Zukauskaite 2014).

Despite the convincing theoretical arguments in favour of cluster policies, there is a lack of empirical evidence that cluster policies in general exert a positive influence on cluster regions (Edler et al. 2013, Uyarra and Ramlogan 2012). These studies show the difficulty of evaluating cluster policies, due to their complexity, high dimensionality, and the time dimension of the policy effects (Rothgang et al. 2017). In this paper, we address these problems by looking at the effects of cluster policies from different

(c) The Author(s). 2017 Open Access This article is distributed under the terms of the Creative Commons Attribution 4.0 International License (http://creativecommons.org/licenses/by/4.0/), which permits unrestricted use, distribution, and reproduction in any medium, provided you give appropriate credit to the original author(s) and the source, provide a link to the Creative Commons license, and indicate if changes were made. 


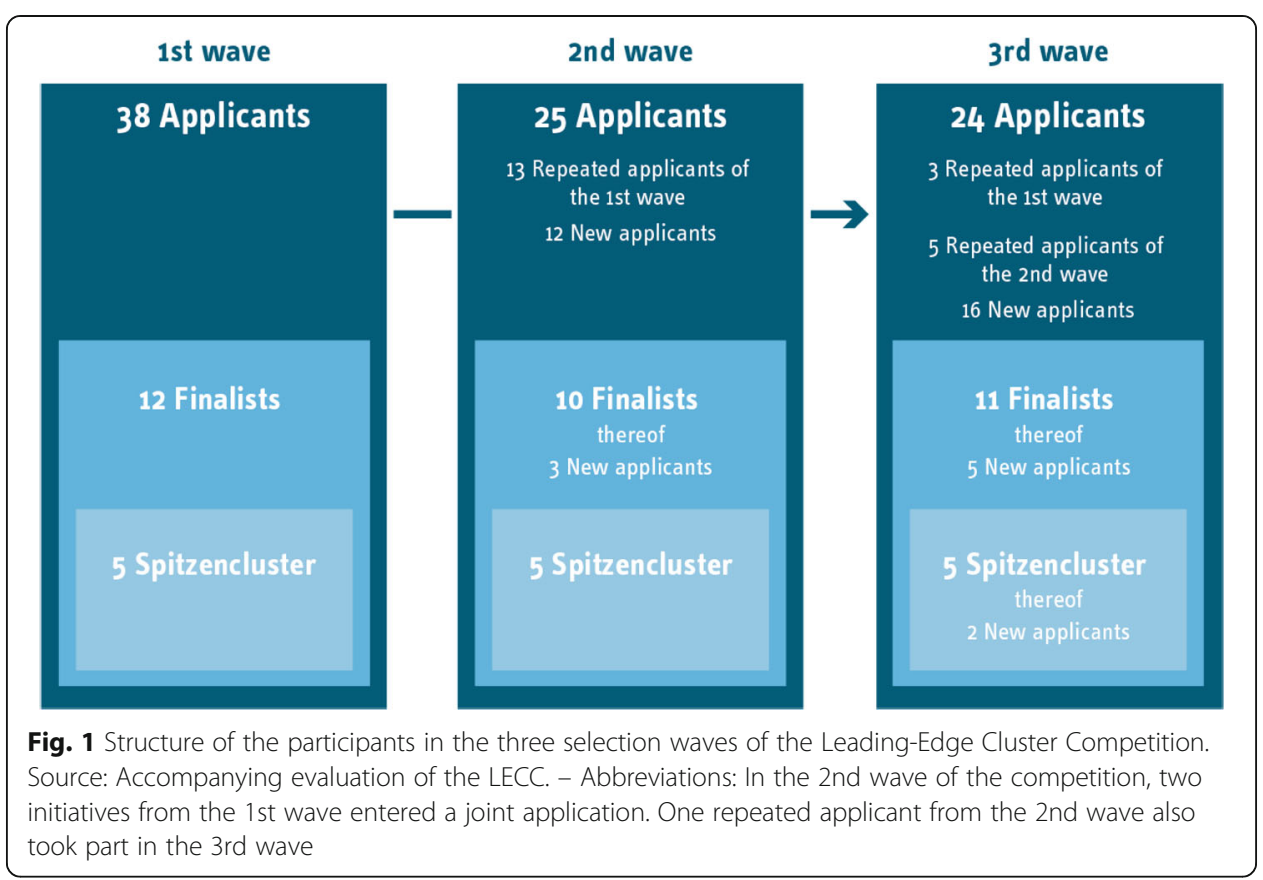

perspectives and on different levels. Specifically, we contribute to our understanding of cluster policies by analyzing procedural aspects of policy implementation, network development, the geographical dimension, and the effects on R\&D activities.

Along these dimensions, we summarize and discuss the main findings of the accompanying evaluation (2008 - 2014) of the German policy programme Leading-Edge Cluster Competition (LECC). The LECC was initiated in 2007 by the German Ministry of Research and Education, continued until 2017, and is up to now the largest federal cluster programme with an overall budget of 600 Mill. $€$.

The paper proceeds as follows: Section 2 provides a detailed account of the LECC, compares its main features with prominent other national and international cluster programmes, and introduces the scheme for our impact evaluation. Section 3 analyses the positioning of the 15 clusters in the international (European) context. Sections 4, 5 and 6 address different outcome dimensions of the LECC, networking, regional impact, and effect on R\&D investment. Section 7 concludes with policy implications.

\section{The Leading-Edge Cluster Competition}

With its Leading-Edge Cluster Competition (in German: Spitzencluster-Wettbewerb; LECC), the Federal Ministry of Education and Research (Bundesministerium für Bildung und Forschung, BMBF) is supporting innovation clusters in a nationwide contest for the first time. In three waves, 15 cluster initiatives were selected and provided with funds to support them on their way to becoming international leaders in their field of technology, or, if they already held such a position, to maintain or expand their lead. Through a sustainable mobilisation of regional economic potential, supporting the subsequent strategic development of Leading-Edge Clusters has the goal of increasing growth, securing or creating jobs and enhancing the attractiveness of Germany as a location for innovation and business. 
The LECC applied a two-step selection procedure supported by a jury. The design of the selection process reflects the goals of the competition and takes into account the requirements for strategic coordination in a multi-stakeholder programme. The selection process was transparent and well communicated. As the feedback by the candidates consistently shows, there was sufficient time to coordinate and prepare the proposals. The selection criteria of the jury are plausible and were implemented adequately using a holistic approach that required the consideration of different dimensions of the programme requirements. The jury was independent and had the opportunity to consult expert advice in decision-making. Figure 1 illustrates the distribution of LECC applicants over the course of the three waves of the LECC competition.

As can be seen, the LECC was successful in motivating new cluster initiatives for the second and third wave of the competition. Moreover, several initially unsuccessful cluster initiatives were able to advance their organisational and strategic development in a manner that they succeeded in a later wave. In the second wave of the competition, only contestants that had already participated in the first wave - some of them in different constellations - were selected. In the third wave, a number of new candidates succeeded.

The evaluation of the LECC selection process also involved a comparison with other, internationally visible technology development programmes of high nationwide importance: the Pôles de Compétitivité in France, the Competence Centers for Excellent Technologies in Austria (COMET), the Centre of Excellence programme (SHOK) in Finland, as well as (on the German state level) the "Cluster Offensive Bayern" (a Bavarian cluster programme).

Like the Pôles de Compétitivité and COMET, the LECC used an application-based approach to select funded initiatives. The regional (political) level also played a certain role during the pre-selection period in these programmes. This regional focus was less pronounced in the Pôles de Compétitivité. The SHOK programme and the Bavarian Cluster Offensive used a top-down approach that was initiated by a comprehensive analysis during the preparatory stage. The evaluation results show that the application procedure is a key factor for the cluster initiatives to focus and constitute their activities. In addition, the results show that the design of the LECC was suitable for achieving the programme's objectives. Like the LECC, the other selected programmes are implemented since several years.

In respect to targets, all these cluster programmes - except for the Bavarian "Cluster Offensive" - focus primarily on research and innovation. A difference between the programmes is that the Pôles de Compétitivité and the LECC do not involve any funding of the underlying cluster management (CM) structures. However, there was no indication that the decision not to allocate funding to CM structures resulted in any disadvantages for either programme. After all, the cluster initiatives in both programmes showed a positive development. Another difference is the funding of infrastructures, which is possible within the Pôles de Compétitivité and SHOK and not in other programmes. All programmes, including the LECC, explicitly support applications for complementary funding on the national or EU level. The funding periods are of similar length and appear to be suitable for achieving the goals of supporting cluster development. 




It is too early to quantify the extent to which the LECC has contributed to strengthening the clusters. However, it is possible to estimate whether the conditions are suitable for the competition to exert a noticeably positive influence in future. To answer this question, the role of Leading-Edge Clusters in their innovation environment was investigated in order to assess their regional and sectoral positioning.

Figure 2 shows the geographic distribution of the 15 Leading-Edge Clusters and roughly 640 cluster initiatives that were identified in Germany at the national or Länder 


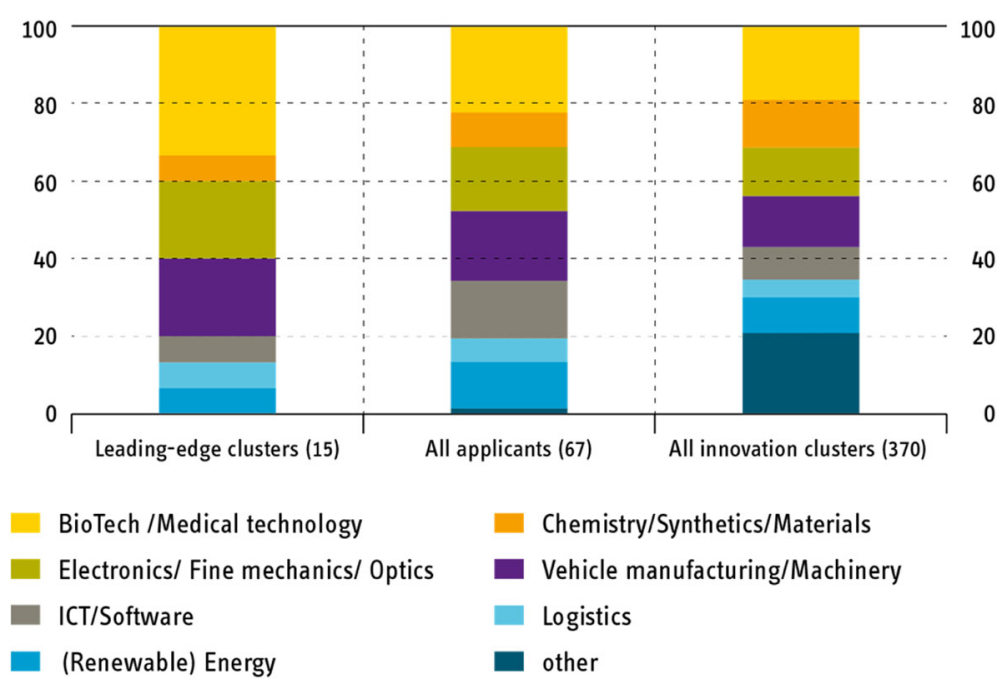

Fig. 3 Industry distribution of the cluster initiatives in comparison. Source: Accompanying evaluation of the LECC, based on an own inquiry (date: November 2013). The LECC had an above average number of applications from cluster initiatives in the fields of biotechnology and medical technology. A possible cause may be that several funding programmes for networks and clusters in this industry have been conducted in the past (e.g. BioRegio), which led to the development of structures that were beneficial in the LECC candidacy. This is true not only for the group of candidates, but even more for successful initiatives: The share of biotechnology and medical technology clusters is even higher in the 15 Leading-Edge Clusters

level by the end of 2013. The sheer number of cluster initiatives, which in almost all cases receive funding, primarily by the states, shows the importance that promoting clusters has gained for innovation policy in Germany. While all the German Länder support clusters, the number of funded clusters and the intensity of support vary considerably. The Leading-Edge Clusters are integrated into existing network and cluster structures that are also supported by the Länder.

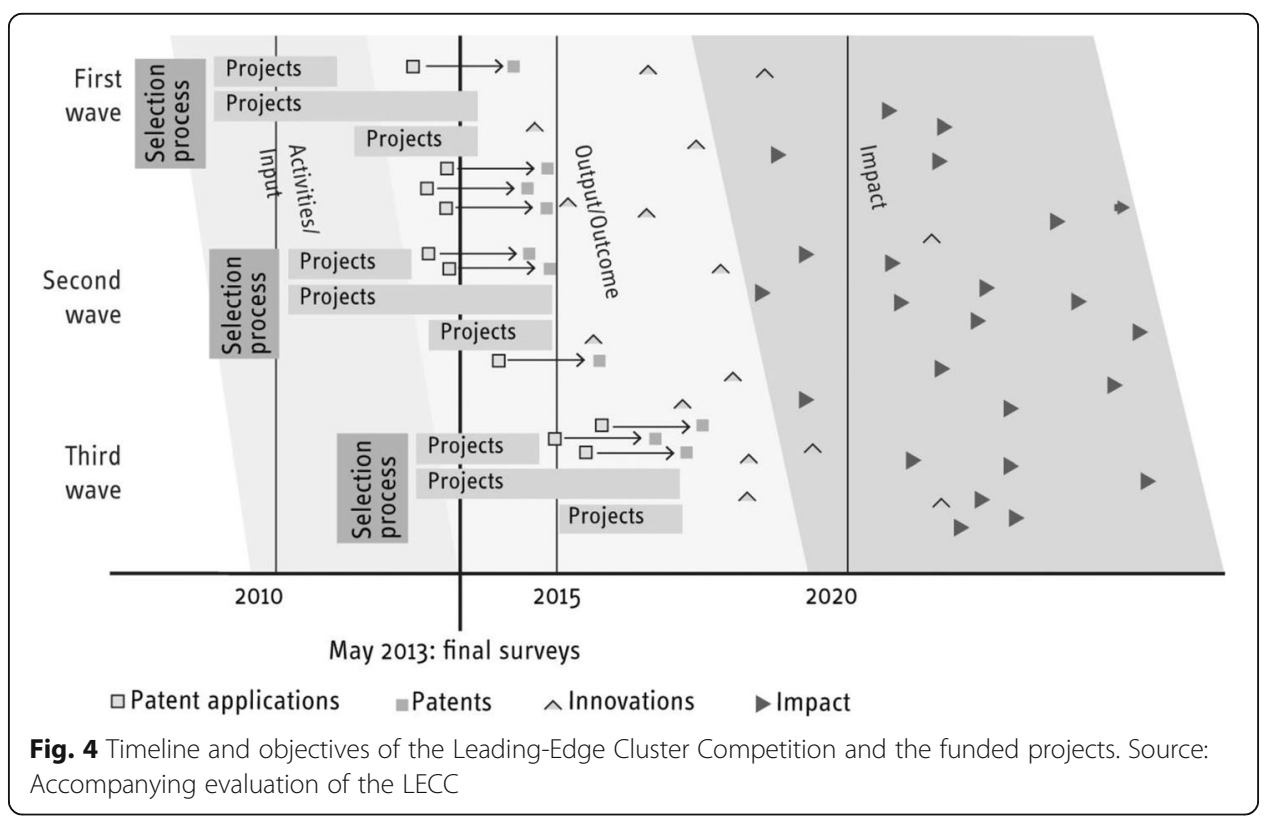


The financial support for Länder clusters is usually for a limited term only, sometimes using financial support from the European Fund for Regional Development (EFRE). Besides other factors, the regional distribution of cluster initiatives results from the regional industrial structure: The more a region is industrialised, the greater the number of clusters initiatives and networks (e.g. in North Rhine-Westphalia and Baden-Württemberg). There is a north-south divide in the distribution of the 15 Leading-Edge Clusters: Nine are located in southern Germany. In addition to the industrial structure, the observed regional differences also reflect the research intensity of the local economy. Finally, the observed differences also depend on the degree to which firms, science and government succeed at joining forces to pursue a common strategy.

The LECC had no restrictions in respect to sectors or technology fields, which is why the Leading-Edge Clusters are rooted in different sectoral innovation environments that exhibit diverse patterns of research and innovation. Figure 3 compares the sectoral distribution of the Leading-Edge Clusters with the distribution of all the participants in the LECC, as well as the entirety of comparable innovation clusters in Germany that were identifiable at the national and Länder levels. The number of innovation cluster initiatives (370) is significantly lower than the total number of cluster initiatives. The latter also includes cluster initiatives that cannot be categorised as innovation clusters due to the industries in which they are active or other characteristics.

The effective dimensions of the LECC are reflected in the funding guidelines for the competition in the form of short-term, midterm and long-term objectives (Fig. 4). The general timeline of the effects outlined here was confirmed by the analysis.

The effects that correspond to these goals range from activities that were initiated (expenditure of additional funds by the stakeholders, networking, knowledge exchange, changes in the $\mathrm{CM}$ processes) to direct and indirect results of the cluster activities (innovation, technology development) to long-term economic effects (increased competitiveness, value-added, employment and wealth). To be certain that the results described here are actually caused by the LECC, it would be ideal to make a comparison with the development of the Leading-Edge Clusters without the LECC. This so-called counterfactual situation is not observable. However, the methods of econometric group comparison analysis make it possible to scrutinize whether an observed effect has actually been caused by the LECC. These methods were used wherever the available data permitted. In many cases where this was not possible, the question whether observed results were caused by the LECC was addressed based on well-founded assessments.

\section{Leading-edge clusters in the international context}

An international comparison showed how Leading-Edge Clusters are rooted in their sectoral innovation systems and displayed their innovative potential. The assessment of this aspect is based on literature research, the examination of patent microdata from research and innovation surveys, and the results of interviews with industry experts, consultants and cluster stakeholders. Even though the industries or sectors as well as the clusters operating in these different environments are usually characterised by a high degree of heterogeneity, they exhibit some predominant and common patterns of research and innovation behaviour.

All Leading-Edge Clusters show strengths and potentials that characterise them as "leading-edge regions" when compared to other international clusters and enable them 


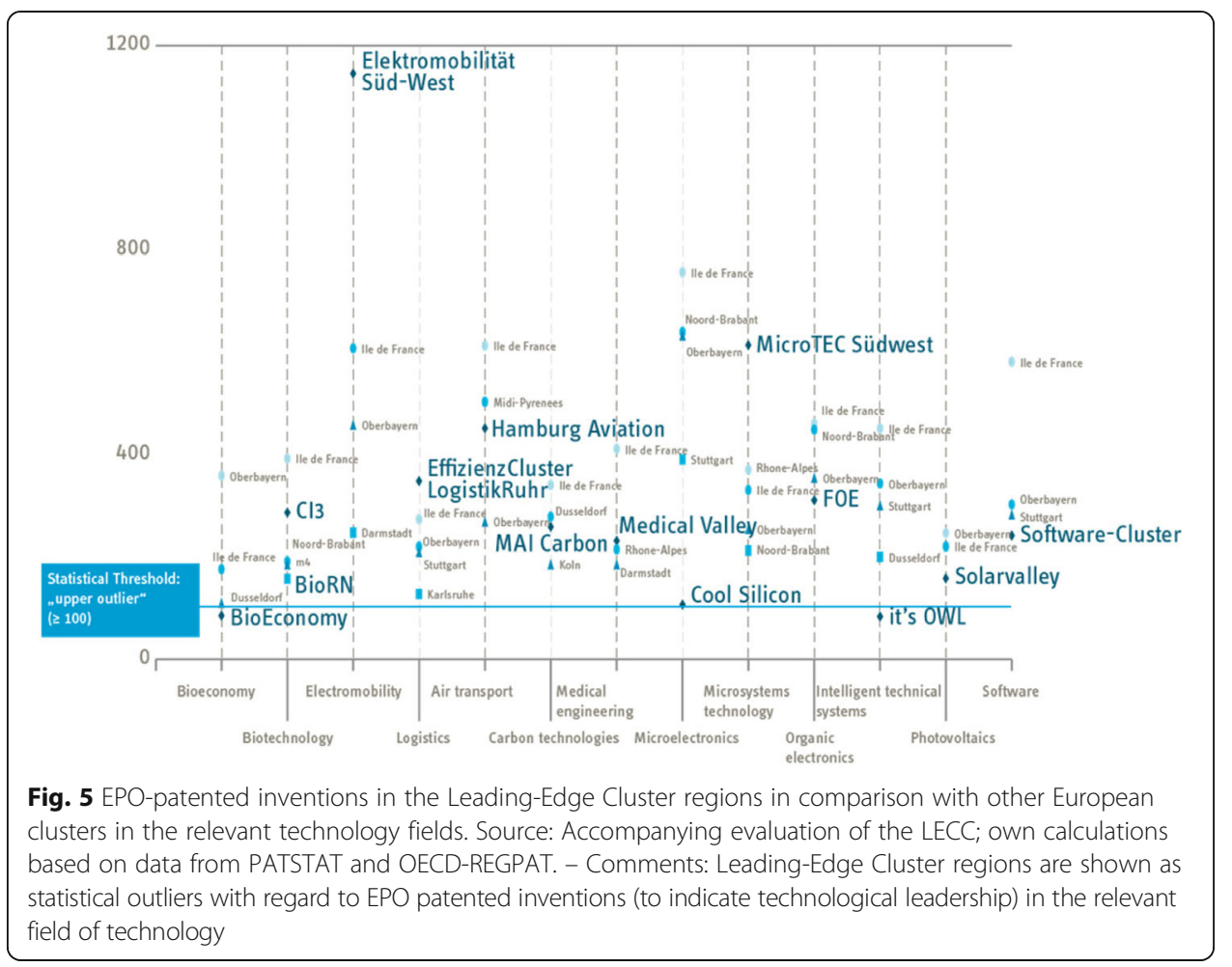

to maintain or expand their position in the international competition. The reasons for this good position are the existing technological strengths and scientific excellence, as well as the presence and regional involvement of globally operating, leading companies (such as in the clusters BioRN, CI3, EffizienzCluster LogistikRuhr, Elektromobilität Süd-West, FOE, Hamburg Aviation, Medical Valley and Software Cluster). In some cases (it's OWL and MicroTEC Südwest), the hidden champions (major firms that attain a leading role in their respective markets) provide the momentum for innovative potential and future competitiveness.

In some fields, in particular biotechnology, innovations are driven primarily by basic research and therefore determined by the quality of key university institutions. In others, such as microelectronics, micro-system technology, medical technology, and intelligent technical systems, internationally renowned research facilities outside of universities significantly determine the positioning of the relevant clusters. Another factor that influences the position of the clusters in the international innovation environment is a core role as a production location (e.g. Cool Silicon, it's OWL and Solarvalley).

Figure 5 shows the technological positioning of various clusters in the European environment. It shows the extent to which the regions of the Leading-Edge Clusters have a position of international leadership in their field of technology with regard to their share of the patent applications at the European Patent Office (EPO). The patent analysis was performed in several steps: By using a combined query for selected classes of patents and technical terms, the number of patent applications for the relevant sector at the EPO was determined and then assigned to regions based on the location of the inventor. 
The statistical definition of an upper outlier was used to define "leading-edge regions". These are regions whose share of inventors is further away from the median than three times the interquartile range. On this basis, European "leading-edge regions" are identified, a group to which the regions of the Leading-Edge Clusters belong.

The analysis shows 12 of the 15 Leading-Edge Clusters as having a position of technological leadership in the European environment. Only the BioEconomy Cluster and it's OWL are slightly below the - albeit very strict - threshold for an upper outlier. Cool Silicon is only slightly above. All other clusters are more or less clearly above this ambitious threshold. Especially Solarvalley has become much more important with regard to patented inventions in recent years and is now clearly above the threshold.

\section{Networking and knowledge exchange in the leading-edge clusters}

With the incentives for networking between regional actors, the LECC supports both the generation and the transfer of knowledge. Within the scope of cluster activities, networking and knowledge exchange may occur as part of the initiated projects, but also through personal contact outside of the project context or during informal meetings. Cooperation in R\&D projects is of central importance in the LECC because it enhances networking relationships, initiates learning processes in the participating organisations, and enables the utilisation of synergies between actors. Learning processes take place through collaboration in projects where different individuals from

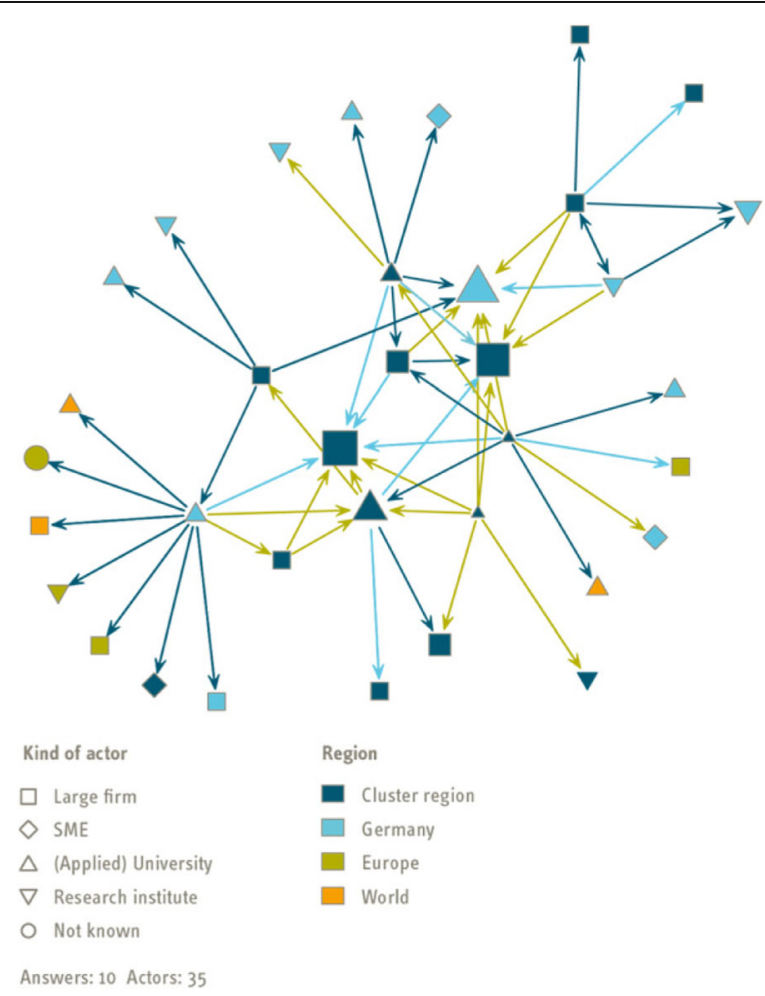

Fig. 6 Cooperation network of the cluster Forum Organic Electronics. Source: Accompanying evaluation of the LECC; written survey of the LECC-funded organisations in 2013. - Comments: The size of the nodes is proportional to the frequency with which an actor is named as the most important cooperation partner. Arrows pointing in the direction of the named cooperation partner; dark blue $=$ not initiated or intensified by the LECC, light blue = intensified by the LECC (existed before 2007), light green = initiated by the LECC (did not exist before 2007) 
companies and public research organisations participate. These individuals proceed to spread knowledge within their organisations.

Such cooperation may either be bilateral or multilateral within larger project consortiums. A cooperation network as studied with the tools of network analysis represents all interconnections constituted by such cooperation. The analysis of networks goes beyond individual collaborative relationships and considers the structure resulting from all bilateral or multilateral cooperative ties so that the structural effects of cluster policies become visible (Giuliani and Pietrobelli 2014).

In order to analyse the impact of the LECC's support on networking and the structure of networks, the cooperation activities related to the LECC were investigated in the context of all partnerships. As a data source, the written surveys of the LECCfunded organisations in the clusters of the first wave of competition in 2011 and 2013 as well as those of the second wave of competition in 2012 were used. Additional information was collected from personal interviews with selected partners in the LeadingEdge Clusters.

Figure 6 exemplifies how this information is utilized to reconstruct network graphs for the collaborative network of the cluster Forum Organic Electronics.

In addition to visualisation, which in this case illustrates the central importance of large corporations in the cluster region, the relational data allows for statistical calculations to describe network structures in the clusters. For example, there are significant differences with regard to size, regional involvement and other structural properties in the R\&D cooperation networks of the ten Leading-Edge Clusters in the first two competition waves.

The stimulus from the LECC had an impact on the intensity and geographic range of networking and changed the centralisation structure of networks. Since the start of the LECC, the intensity of network cooperation increased in all clusters of the first and second competition waves, which became evident as collaborative activities significantly

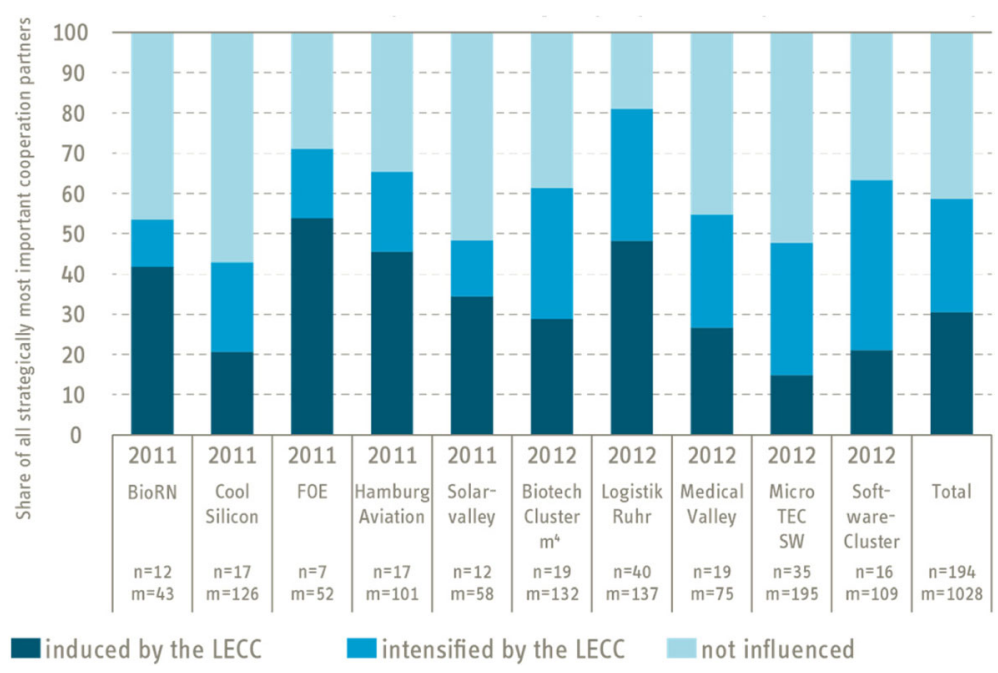

Fig. 7 Share of LECC-influenced relationships in all strategically important cooperation relationships. Source: Accompanying evaluation of the LECC; written survey of LECC-funded organisations in 2011 and 2012. - Comments: $n=$ Number of responses; $m=$ Number of identified relationships within the cooperation network 


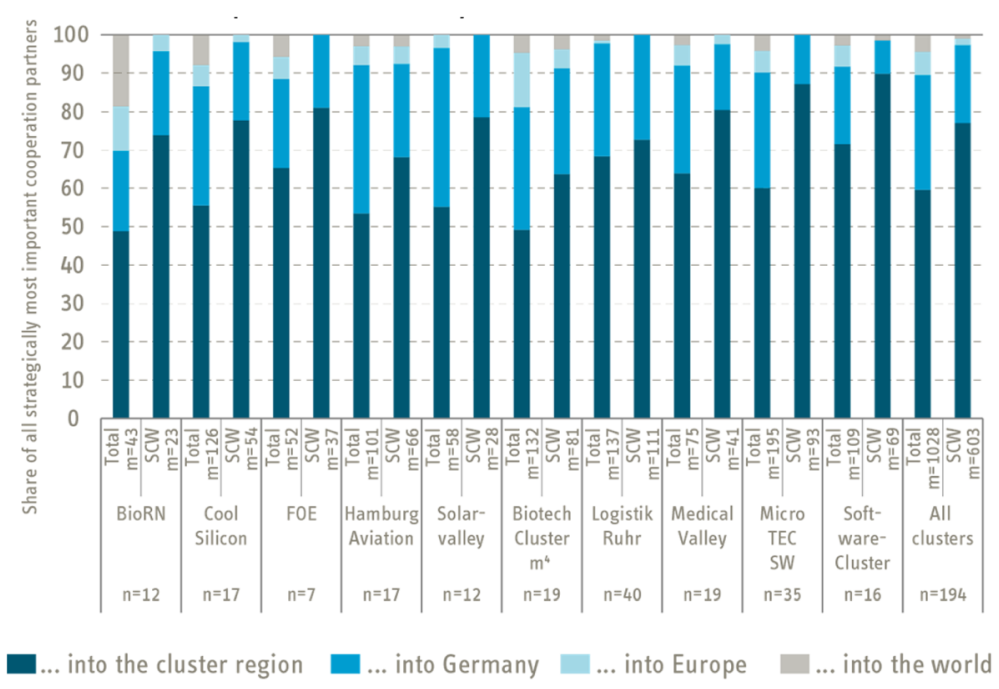

Fig. 8 Geographic distribution of strategically important collaborative relationships - comparison of the entire network of cooperation influenced by the LECC. Source: Accompanying evaluation of the LECC; written survey of LECC-funded organisations in 2011 and 2012. - Comments: $n=$ Number of responses; $m=$ Number of identified relationships within the cooperation network

increased. This is primarily due to improved awareness of potential partners as consequence of the LECC. Especially for the businesses among the LECC-funded organisations, the importance of cooperation in their overall $R \& D$ activities has increased. $R \& D$ cooperation between competitors occurred much more frequently than on the national average, as a comparison with data from the Mannheim innovation panel shows.

The LECC initiated new R\&D partnerships and intensified existing contacts. A substantial mobilisation effect, extending beyond the LECC-funded cooperation projects, can be observed in the initiated relationships (Cantner et al., 2015). Figure 7 shows the influence of the LECC on relationships in R\&D cooperation networks in Leading-Edge Clusters.

In some of the clusters (EffizienzCluster LogistikRuhr, FOE, Hamburg Aviation), almost one half of all relationships were initiated by the LECC. In the clusters MicroTEC Südwest and Cool Silicon, the LECC appears to primarily intensify existing relationships. These differences between clusters depend primarily on whether there were preexisting, intensive R\&D networks before the LECC or if these networks had to be built from scratch. The latter was the case in the Effizienzcluster LogistikRuhr, where many stakeholders had not been involved in R\&D activities before the competition.

The number of cooperations that bridge the gap between science and business has increased. However, the relative importance of cooperation among public research organisations or between public research organisations and businesses has remained almost unchanged. As intended by the LECC, in many cases the competition was used to build new cooperative relationships within the Leading-Edge Clusters. Numerous new collaborations in project consortiums were initiated. However, these partners had in general not been completely unknown to each other.

The LECC also had the desired impact on the geographic range of networks. However, there are considerable differences with regard to the degree of regional 
networking and international engagement between the clusters (Fig. 8). For example, the Effizienzcluster LogistikRuhr and the Software Cluster show a comparatively high degree of networking within the cluster region. Hamburg Aviation and Solarvalley are primarily embedded on the national level, whereas the stakeholders in the two biotechnology clusters BioRN and Biotech Cluster $\mathrm{m}^{4}$ are involved in markedly international networks. Despite these differences, the focus of all $R \& D$ cooperation networks lies within their cluster region. The R\&D cooperations which were initiated by the LECC show a stronger regional element than other partnerships. This result illustrates that the LECC contributed to opening and exploiting regional innovative potential. For the successful clusters of the first wave, Cantner et al. (2015) observe differential developments regarding clusters' spatial embeddedness with two additional years of observation. Some clusters tend to increase their localisation, whereas others increase their connectivity to international partners.

An examination of the centralisation structure of the networks shows that relationships formed during the early stages of the LECC are often more focused on key stakeholders (usually large corporations or public research organisations). Analyses of a change in centrality during later stages show that actors which were already central in the previous period and large firms benefit most, whereas universities and public research institutes become less central within the R\&D networks (Cantner et al., 2015). However, if the authors control for policy measures such as the number of funded projects and the total amount of funding renders all other variables insignificant. Apparently, those who benefit most from funding, also benefit most in terms of increased embeddedness in the network. During the LECC, these stakeholders have established themselves as important partners and made essential contributions for the technological and organisational development of the clusters. This development is understandable for competitions such as the LECC, because the common cluster strategies are usually developed under the leadership of a relatively small group of renowned and technologically competent actors that subsequently participated in the LECC-funded projects. Many SMEs used the LECC as an opportunity to build relationships with large corporations that would have been difficult to access otherwise. The establishment of long-term R\&D contacts is especially important for SMEs in order to build relationships for innovation and assist the effective commercial exploitation of their developments.

The results of the analysis also show that some large corporations contact companies and research institutes for specific purposes, for example to solve current research problems or to benefit from their competence in the medium or long term. While excessive concentration of the networks on a few key actors may harbour a risk of becoming too dependent on their development, this study has found no indication of such a risk in practice.

All in all, the LECC's short-term goal of intensifying or enhancing the networking between innovative stakeholders in the cluster regions has been achieved. To what extent this enhanced networking will have a long-term impact on successful innovation depends on whether the cooperation with local or supra-regional partners will remain at a high level beyond the funding period. The results of the investigation show that many relationships that were initiated are intended for long-term cooperation and should therefore have a sustainable impact on the cluster networks. 


\section{Regional impacts of the leading-edge cluster competition}

One of the stated goals of the LECC is to generate long-term value through the exploitation of regional innovation potentials. In this context, a main focus is on the analysis of mobilisation processes at the core and in the environment of the clusters. Specifically, the significance of regional location factors, of geographical proximity to R\&D partners as well as first observable and potential future impacts of the competition on the cluster regions were investigated. At the moment, the immediate effects of the competition in the Leading-Edge Clusters can primarily be observed (input and activity effects, partially first outputs) while outcomes and economic impact will rather be observable in future years. Hence, it was necessary in a first step to categorise the clusters with regard to the relevance of technological and economic location factors for the LECC-funded organisations.

The investigation of the regional impacts of the LECC incorporates the information from the written survey of LECC-funded organisations, the interviews with the CMs and cluster actors and the findings from the analysis of the sectoral innovation systems and networking. The results of the surveys were primarily evaluated by means of descriptive analyses. In order to account for the heterogeneity of the responses between the clusters, the correlations between cluster specifics and response behaviour were estimated by means of multivariate regression methods.

The analysis of the relevance of regional location factors considers general economic conditions for cluster activities, for example regional labour and sales markets, the technological conditions, as well as geographical proximity to R\&D partners as a determinant for knowledge exchange. With regard to regional location factors, actors in all clusters selected in the first two competition waves rate the local supply of highly skilled employees (college and university graduates) as most important, followed by the availability of medium skilled workers. This underpins the importance of cluster activities to qualify current and future employees. Differences between the clusters with regard to the importance of the regional labour market can be explained by differences in the composition of actors or differences in the technological focus.

Compared to the local labour market, the local sales market is of minor importance for the LECC-funded organisations in the clusters of the first two competition waves. Clusters with a comparatively high number of public research organisations show higher ratings for this item than other clusters. This can be explained by the fact that public research organisations tend to acquire their third-party funds locally - at least when it comes to third party funding by companies - while companies, even those with important local customers, operate to a greater extent on supra-regional markets. Industry-specific factors play a crucial role for the importance of the regional sales markets. For example, the revenues of Hamburg Aviation and Medical Valley are more concentrated on large local companies than in the case of the Biotech Cluster $\mathrm{m}^{4}$.

Regarding their technological environment, LECC-funded organisations mostly agree that, though geographic proximity facilitates $R \& D$ cooperation, it is not a necessary requirement for its success (Hinzmann et al. 2017). 90\% of the LECC-funded organisations consider cooperation with geographically close partners as less cost-intensive. $80 \%$ think that geographical proximity facilitates information exchange. $35 \%$ of the LECC-funded organisations regard geographical proximity as a central precondition for the success of the cooperation. However, technological aspects and the partners' 


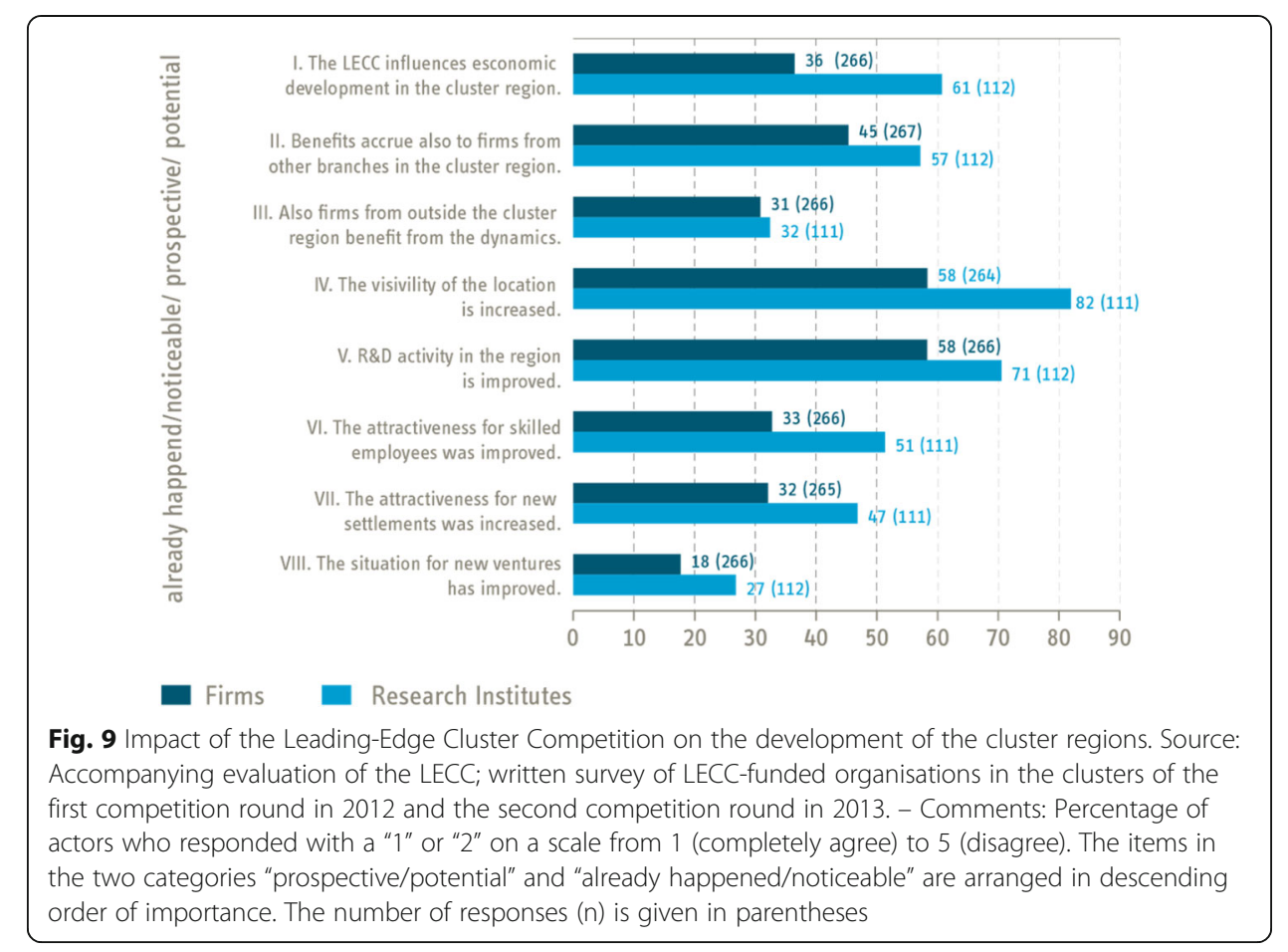

qualification play a superior role for the selection of cooperation partners than their geographical location. Accordingly, LECC-funded organisations concentrate their search for R\&D partners rather on the national than on the regional level. Furthermore, organisations in Leading-Edge Cluster projects report higher satisfaction with the cooperation, when the partners have already worked before in other contexts. All in all, the analyses show that the requirements of Leading-Edge Cluster actors in respect to their regional environment are driven by their orientation towards knowledge-intensive industries. The supply of qualified employees and the requirements for regional $R \& D$ cooperation are of greater importance than the proximity to customers or suppliers.

According to the LECC-funded organisations in all clusters of the first two competition waves, the LECC has already triggered or will trigger regional impetuses. Figure 9 shows the assessment of potential or observable effects of the LECC separately for firms or research institute.

$44 \%$ of the LECC-funded organisations expect that the success of the Leading-Edge Cluster will have a noticeable effect on the development of the cluster region. The largest effects of the LECC can be found in the increased visibility of the location and the improved regional $R \& D$ activity. Public research organisations exhibit a significantly more positive view - probably because of the greater importance of external funds for research and a resulting different view on the regional impacts.

The assessment of the impacts varies considerably between the clusters. This can mainly be explained by the clusters' composition, in particular the ratio of public research organisations to companies. Clusters with a high share of public research organisations have a considerably more positive view of the effects. Furthermore, geographically extended clusters, as well as clusters with a large number of LECC-funded organisations, appear on average to have a more sceptical view of the competition's effects on the region. A possible 
cause may be that the close geographical proximity between partners in more concentrated clusters fosters the exploitation of synergy effects.

At the moment, several types of regional impulses of the LECC can already be observed, primarily an improved visibility of the Leading-Edge Cluster regions and enhanced regional R\&D activity. The actor interviews yielded that collaboration in the joint projects has improved the innovative climate and resulted in the formation respectively in an advancement of a common culture of innovation. While the stimuli from the LECC may not be able to fully compensate for critical economic trends, the LECC-funded projects still laid the basis for future increases in the generation of regional value added. By intensifying and initiating further R\&D cooperation, the LECC is primarily effective in earlier stages of the value chain. Hence, its effects on growth and employment are not noticeable in the short run.

\section{Effects of the leading-edge cluster competition on R\&D funding}

From an evaluation perspective, it would be desirable to know whether public $R \& D$ funding has a causal effect on private $R \& D$ activities. However, the time lags inherent in funding effects, the complexity of diffusion mechanisms of cluster policies, and the resource intensity in the application of available methods to identify causal policy effects impede efforts to quantify the actual policy effects. Against that backdrop, our goal was to identify the causal effects of the LECC on firm-level $R \& D$ activities (both $R \& D$ expenditure and $R \& D$ personnel) and compare the effects to other R\&D funding programmes. ${ }^{1}$

Starting point of our analysis were the results of prior studies on the causal effects of $R \& D$ funding and cluster policies. The effects of public R\&D promotion have been analyzed extensively with no clear evidence on the effects of the policy measures arising (see e.g. David et al. 2000, Czarnitzki, Ebersberger and Fier 2007). Especially it remains unclear whether the promotion of private R\&D in public programmes leads to no crowding out, partial or full crowding out (Duguet 2004, Czarnitzki and Fier 2002 and Wallsten 2000). At the same time, existing quantitative evaluations provide no clues as to what effects to expect from cluster policy measures. This is due to different funding mechanisms, but also different target variables of the evaluations such as innovation (Falck et al. 2010 for cluster policies in Bavaria and DAMVAD 2011 on a Danish programme) or employment/turnover (Martin et al. 2011). While both, the Bavarian and Danish programme are not focusing on R\&D funding, the predecessor of the pôles de compétitivité in France, which was analyzed by Martin et al. (2011), promoted the development of clusters affected by structural change. While Falck et al. (2010) and the study on Denmark (DAMVAD 2011) found that these cluster programmes were successful in promoting innovation activities in the clusters that were scrutinized, Martin et al. (2011) could not identify effects of cluster policy whatsoever.

In the LECC evaluation, we estimated the "average treatment effect on the treated" (ATT) as a measure of the impact of R\&D funding on R\&D activities of the funding recipients. In order to assess the programme effect, we had to rely on a nonexperimental approach that has previously been applied in similar studies (e.g. Blundell and Costa Dias 2000, Smith and Todd 2005). This hybrid matching approach combines propensity score matching with the application of a Mahalanobis distance estimator to account for few rather important variables (see e.g. Lechner 1998, Almus and 
Czarnitzki 2003). Based on this approach, we estimate the ATT by calculating a difference-in-difference-estimator. ${ }^{2}$ In order to reach unbiased estimates of the effects of cluster policy, we used nearest neighbor matching with replacement (making it possible to match single non-treated firms with more than one treated). This allows to identify one non-treated firm for each treated with the closest probability of mode change (for details on the approach see Lechner 2001). ${ }^{3}$ By applying this method, we are able to address the counterfactual question: "what would have been the outcome for LECC program participants if they had participated in other funding schemes?” We compare firms funded by the LECC both to firms that did not receive funding and firms that have been funded in other programmes.

The analysis is based on a data set that combines four sources: (1) data from the R\&D statistic of the business sector collected by the SV Wissenschaftsstatistik (a biannual survey of all R\&D active firms in Germany). We use data for the years 2007, 2009, and 2011. (2) Data from the Dafne database (release February 2012). (3) Information on R\&D funding and funded firms from the PROFI database on R\&D project funding by the Federal Government. (4) Data from our own surveys of firms promoted by the LECC.

Table 1 shows core results of our regression analysis. We find that R\&D expenditures of firms funded by the programme are significantly higher compared to non-funded firms. On average, R\&D expenditures of funded firms increased by $45 \%$, while it decreased by about $14 \%$ for the matched non-funded firms. The difference is even larger when looking at the effect of the programme on SMEs (an increase of 58\% for funded SMEs was estimated compared to a decrease of $20 \%$ in the comparison group). When looking at different measures of $R \& D$ intensity, we find that the change in $R \& D$ expenditure per employee is significantly higher for funded firms than for non-funded firms (both for all firms and SMEs).

Similar results are obtained when looking at the firms of the 2nd wave for which an additional pre-funding period can be used to account for unobservable firm characteristics as project funding started in 2010.

When comparing firms that receive LECC funding with firms that have been funded by other programmes, we find that LECC funding leads to a significant increase in some but not all measures (such as external R\&D expenditures as a share of firm turnover, not displayed in Table 1). This result has also been confirmed by individual observations at the firm level with some firms being more prone to participate in other programs due to their participation in the LECC: Furthermore, our results show that the LECC has especially influenced the behavior of SMEs. The growth of R\&D expenditure is larger for SMEs funded by the LECC than for SMEs funded otherwise. This is consistent with our observation that the program made it easier for SMEs to gain access to established R\&D networks.

Based on our results, it was possible to analyze the degree of additionality of LECC funding which is important to understand how firms react to public funding and to assess the effect of R\&D subsidies on funded firms. In this analysis, we have to bear in mind that funded firms are required to complement public funding with the same amount of internal R\&D funding. However, it is not clear ex ante whether the additional funds are provided by increasing the internal R\&D budget or by shifting $R \& D$ funds from other project activities. Thus, we distinguish full additionality (i.e. the increase in overall $R \& D$ expenditure relates to the amount of public funding plus the 
Table 1 Group comparison after matching (LECC funded vs. non-funded, mean values)

\begin{tabular}{|c|c|c|c|c|c|c|}
\hline Outcome variable & LECC-funded & Non-funded & $A T_{\mathrm{DiD}}$ & t-test & & $\#$ \\
\hline \multicolumn{7}{|l|}{ All companies (2007-2011) } \\
\hline CGR R\&D expenditures & $0.45^{\mathrm{a}}$ & -0.138 & 0.588 & 3.39 & \multirow{2}{*}{$\begin{array}{l}* * * \\
* * * \\
*{ }^{* * *}\end{array}$} & 135 \\
\hline CGR internal R\&D expend. & 0.403 & -0.122 & 0.525 & 2.99 & & 110 \\
\hline CGR external R\&D expend. & 0.42 & 0.565 & -0.145 & 0.43 & \multirow[b]{2}{*}{${ }^{* *}$} & 109 \\
\hline CGR R\&D personnel & 0.279 & 0.022 & 0.257 & 2.14 & & 72 \\
\hline$\Delta R \& D$ exp./employees & 5.681 & -0.229 & 5.91 & 2.56 & ** & 134 \\
\hline$\Delta R \& D$ exp./turnover & 0.143 & -2.555 & 2.698 & 1.22 & & 135 \\
\hline$\Delta$ internal R\&D exp./turnover & -1.67 & -2.204 & 0.534 & 0.25 & & 110 \\
\hline$\Delta$ external R\&D exp./turnover & 1.26 & -0.35 & 1.61 & 1.85 & * & 110 \\
\hline \multicolumn{7}{|l|}{ SME only (2007-2011) } \\
\hline CGR R\&D expenditures & 0.576 & -0.197 & 0.774 & 3.74 & \multirow{2}{*}{$\begin{array}{l}* * * \\
* * *\end{array}$} & 80 \\
\hline CGR internal R\&D expend. & 0.552 & -0.217 & 0.77 & 3.36 & & 57 \\
\hline CGR external R\&D expend. & 0.624 & -0.26 & 0.884 & 2.74 & *** & 57 \\
\hline CGR R\&D personnel & 0.295 & -0.064 & 0.359 & 2.63 & \multirow{2}{*}{$\begin{array}{l}* * * \\
* * * \\
* *\end{array}$} & 48 \\
\hline$\Delta R \& D$ exp./employees & 6.155 & -3.269 & 9.424 & 2.81 & & 79 \\
\hline$\Delta R \& D$ exp./turnover & -0.943 & -4.941 & 3.998 & 1.12 & & 80 \\
\hline$\Delta$ internal R\&D exp./turnover & -4.273 & -5.096 & 0.823 & 0.22 & \multirow[b]{2}{*}{$* *$} & 57 \\
\hline$\Delta$ external R\&D exp./turnover & 1.901 & -1.183 & 3.084 & 1.96 & & 57 \\
\hline
\end{tabular}

Discrete change $(\Delta)$ or continuous growth rate (CGR) of mean values. ${ }^{\text {a }}$ The value 0,45 means an $45 \%$ increase. AT ${ }_{\text {DiD: }}$ : difference between mean values in column 2 and 3 . \# number of observations. ${ }^{*} p<0.10,{ }^{* *} p<0.05,{ }^{* * * *} p<0.01$. For a more detailed description of the method and variables see Engel et al. (2017)

same amount of private funding) and weak additionality (overall firm-level R\&Dexpenditure increases by the amount of public funding). While full additionality is aspired by public authorities, weak additionality is also allowed by the programme requirements. In turn, not even finding weak additionality, meaning that public funding crowds out private $R \& D$, would not be in accordance to the programme targets. Our results show no indications for crowding out on average when we look at all funded firms. We find evidence of weak additionality on average which means that on average private $R \& D$ expenditures have increased slightly. The leverage effect of $R \& D$ funding is higher for SMEs than for large firms, so that the increase in $R \& D$ expenditure excels the amount of public funding for SMEs (for more details see Engel et al. 2017).

The results from our econometric calculations with respect to the leverage effect of $R \& D$ expenditures are consistent with evidence based on qualitative analysis from expert interviews with representatives from cluster firms. These interviews show that differences in the leverage effect of $R \& D$ financing between SMEs and large firms might be due to $R \& D$ budgets of large firms that are in many cases fixed in the medium term. At the same time, SMEs often have the opportunity to adapt their R\&D budget to the project situation, leading to a larger increase in $R \& D$ expenditure as additional funds are obtained from government funding.

Firm interviews also showed marked differences between firms in the leverage effects of government R\&D funding. Some firms substantially increased internal funding as they used their participation in the LECC to build up their R\&D activities (in single cases from scratch). This is, however, not the case for the average firm. In addition, our 
interviews show that the estimated quantitative leverage effect represents only one aspect of the leverage of $R \& D$ funding. As project funding is mainly used for cooperation projects, new ideas created in the projects have the possibility to diffuse between different firms and entail additional medium-and long-term effects.

Finally, the question arises what we can learn from this analysis. We can see that public promotion programmes can increase $R \& D$ activities of business firms. At the same time, we have to bear in mind that increasing $R \& D$ activities is only one of several targets that have to be regarded. This becomes clear when we assess the consequences of the more pronounced leverage effect of the LECC for SMEs than for large firms. One could argue that technology policy should concentrate its effort more on SMEs. However, there are also arguments to the contrary. For the network development of SMEs, which is another programme target, it is often important that also large firms are part of the networks because the SMEs depend on these firms as customers or partners in $R \& D$ cooperation. In addition, cluster programmes try to promote the knowledge transfer between firms. Again, it is important for the successful use of the knowledge to give impulses for projects that involve both SMEs and large companies.

\section{Clusters and innovation policy: Findings and recommendations}

The experiences from the LECC - as well as from other, similar funding programmes in Germany and other countries - can be used for the design and execution of future funding activities. This chapter tries to determine the prerequisites for the success of cluster initiatives. On this basis, recommendations are made for the remaining funding duration of the LECC until 2017, for future cluster and network funding, and for future innovation policies. Wherever possible, the findings of the accompanying evaluation were reflected against a background of existing cluster research and other evaluation studies. There was a high degree of correspondence in many points, as well as some new aspects that are not to be found in the literature (yet).

The "functioning" of technology-oriented cluster initiatives depends primarily on the following factors:

- Technology oriented clustered initiatives can only be successful if they have a critical mass of existing technological and innovation potential to build on already when the initiatives are constituted. If that is the case, the programme may be able to benefit from windows of opportunity that arise not just in early development stages of entirely new technologies, but also in established industries. This happens e.g. when comprehensive technological changes take place or when new challenges arise that require a reconfiguration of known technologies and production factors.

- For the success of cluster initiatives, an assertive cluster organisation represented by suitable cluster managers is indispensable. The cluster organisation and the CM at its core usually need some time in their constitution phase before they are fully functional. Like their corresponding industries, clusters are subject to medium and long-term structural changes. These changes force cluster initiatives to readjust their orientation from time to time and develop their organisations further. In the long run, these cluster institutions should therefore be seen as temporary intermediaries that may be replaced with new structures as this is appropriate. 
- Cluster initiatives are based on exploiting the benefits of geographic proximity. For innovative clusters and in consideration of today's transportation and communication technologies, the word "proximity" can be interpreted pragmatically. The importance of geography varies considerably between the participants of the LECC. In some cases it provides a point of identification that contributes to the mobilisation of regional stakeholders and resources. In other cases it is the result of past developments and taken for granted. In yet other cases, geographic proximity does not play a significant role for joint R\&D activities. A continuous exchange between R\&D employees with different qualifications in one place can be helpful for developing innovations, but is it not a necessary prerequisite for collaboration within a cluster.

- On one hand, the success of cluster initiatives depends on cluster-internal factors. Within limits, cluster organisations are able to compensate for and actively respond to interference from the environment. They are successful especially when sufficient technological and innovation potential is available, when joint activities can be advanced within the cluster organisation, and when positive effects can be achieved by a close regional exchange between cluster stakeholders. On the other hand, environmental factors also play a role for cluster development, in particular international market events as well as framework conditions and their changes (e.g. when certain technological problems cannot be solved or when market constellations change). Such events may necessitate changes in the cluster strategy or, in extreme cases, render the objectives of the cluster organisation obsolete, such that responsiveness and adaptability are required.

The funding of cluster and network initiatives is becoming an increasingly important instrument of innovation policy. Cluster funding addresses technology-political constellations which are characterised by the following factors:

- The development of technologies to be funded is marked by a spatial agglomeration of relevant companies and public research organisations.

- The addressed technologies are at a stage where a technological breakthrough is to be expected in the foreseeable future.

- The clusters to be funded exhibit a critical mass of relevant innovation capacities that may be expected to play a major role with regard to the development of the relevant technologies or industries in the future.

- The cluster initiative to be funded is supported by strong commitment of the stakeholders it represents.

- The technologies and industries in question have significant importance for the total economy.

If one or several of these prerequisites are not fulfilled, then cluster funding is not advisable, or at least not at the federal level. If from the perspective of the total economy there is still a need for funding, then this should be covered by other instruments of research and innovation policy, such as thematic technical programmes, network funding or funding for R\&D cooperation. The instrument of cluster funding is therefore by no means a panacea to solve all conceivable problems of technology policy. Quite the 
contrary: Excessive or even indiscriminate use would necessarily result in a devaluation of the instrument. Regarding the funding of Leading-Edge Clusters, the concept of the LECC was designed in a way that the above prerequisites are all fulfilled. The findings of the accompanying evaluation also confirm that the basic concept of the competition has been implemented as intended.

\title{
Endnotes
}

${ }^{1}$ For a more detailed discussion of the econometric analysis see Engel et al. (2017).

${ }^{2}$ For an application to evaluate labour maket policies see Heckman et al., 1998, for an application to estimate the effect of R\&D promotion see Czarnitzki, Ebersberger and Fier 2007.

${ }^{3}$ Software package psmatch2 in STATA 12 was used (see Leuven and Sianesi 2003).

\section{Acknowledgements}

We acknowledge the constructive discussions with Dr. Verena Eckl, Dr. Werner Friedrich, Dr. Bernhard Lageman, Christian Morgenstern, Dr. Tina Wolf, the members of the DFG GRK 1411 The Economics of Innovative Change, as well as with colleagues at RWI in Essen, at ISG in Cologne, at Joanneum Research Graz, and at the Friedrich Schiller University Jena.

\section{Funding}

Bundesministerium für Bildung und Forschung, Begleitende Evaluierung des Förderinstruments "SpitzenclusterWettbewerb" des BMBF.

Deutsche Forschungsgemeinschaft, DFG-GRK 1411 The Economics of Innovative Change.

\section{Authors' contributions}

Section 1: Conception: MR and JD; finalization: all. Section 2: Conception: MP and EL; finalization: all. Section 3: Conception: UC, HG, and ST; finalization: all. Section 4: Conception: UC, HG, SW and AS; finalization: all. Section 5: Conception: MR, JD, and DE; finalization: all. Section 6: Conception: MF and AS; finalization: all. All authors read and approved the final manuscript.

\section{Competing interests}

The authors declare that they have no competing interests.

\section{Publisher's Note}

Springer Nature remains neutral with regard to jurisdictional claims in published maps and institutional affiliations.

\begin{abstract}
Author details
${ }^{1}$ RWI Leibniz Institute for Economic Research, Hohenzollernstraße 1/3, 45128 Essen, Germany. ${ }^{2}$ ISG - Institut für Sozialforschung und Gesellschaftspolitik GmbH, Weinsbergstraße 190, 50825 Köln, Germany. ${ }^{3}$ JOANNEUM RESEARCH Forschungsgesellschaft mbH, Leonhardstraße 59, $8010 \mathrm{Graz}$, Austria. ${ }^{4}$ Friedrich Schiller University Jena, Lehrstuhl für WWL/Mikroökonomik, Carl-Zeiß-Strasse 3, 07743 Jena, Germany. ${ }^{5}$ University of Applied Sciences Stralsund, Stralsund, Germany.
\end{abstract}

Received: 3 July 2017 Accepted: 28 July 2017

Published online: 22 September 2017

\section{References}

Allen, R.C., (1983). Collective invention. Journal of Economic Behavior \& Organization, 4(1), 1-24.

Almus, M., \& Czarnitzki, D. (2003). The effects of Public R\&D Subsidies on Firms' innovation activities: The case of eastern Germany. Journal of Business and Economic Statistics, 21(2), 226-236.

Blundell, R., \& Costa Dias, M. (2000). Evaluation methods for non-experimental data. Fiscal Studies, 21(4), 427-468.

Brenner, T., \& Schlump, C., (2011). Policy Measures and their Effects in the Different Phases of the Cluster Life Cycle. Regional Studies, 45(10), 1363-1386.

Cantner, U., H. Graf, S. Toepfer, Structural dynamics of innovation networks in German leading-edge clusters, Jena economic research papers, \# 2015-026, 2015.

Czarnitzki, D., Ebersberger, B., \& Fier, A. (2007). The relationship between R\&D collaboration, subsidies and R\&D performance: Empirical evidence from Finland and Germany. Journal of Applied Econometrics, 22(7), 1347-1366.

Czarnitzki, D., \& Fier, A. (2002). Do innovation subsidies crowd out private investment? Evidence from the German service sector. Konjunkturpolitik - Applied Economics Quarterly, 48, 1-25.

DAMVAD (2011). The Impact of Innovation Networks in Denmark - An Impact Study on Behaviour and Possible Economical Effects, Project report. Kopenhagen.

David, P. A., Hall, B. H., \& Toole, A. A. (2000). Is Public R\&D a complement or a substitute for private R\&D? Research Policy, 29, 497-529. 
Duguet, E. (2004). Are R\&D subsidies a substitute or a complement to privately funded R\&D? An econometric analysis at the firm level. Revue d'économie politique, 114(2), 245-274.

Engel, D., Eckl, V., \& Rothgang, M. (2017). R\&D funding and private R\&D: empirical evidence on the impact of the leading-edge cluster competition. The Journal of Technology Transfer, doi:10.1007/s10961-017-9609-5.

Falck, O., Heblich, S., \& Kipar, S. (2010). Industrial innovation: Direct evidence from a cluster-oriented policy. Regional Science and Urban Economics, 40, 574-582.

Fier, A. \& Harhoff, D. (2002). Die Evolution der bundesdeutschen Forschungs- und Technologiepolitik: Ruckblick und Bestandsaufnahme. Perspektiven der Wirtschaftspolitik, 3, 279-301.

Freeman, C., (1991). Networks of innovators: A synthesis of research issues. Research Policy. 20(5), 499-514.

Giuliani, E., \& Pietrobelli, C. (2014). Social Network Analysis Methodologies for the Evaluation of Cluster Development Programs (Papers in Innovation Studies). Lund University, CIRCLE - Center for Innovation, Research and Competences in the Learning Economy. Retrieved from https://ideas.repec.org/p/hhs/lucirc/2014_011.html.

Heckman, J. J., Ichimura, P., \& Todd, H. (1998). Matching as an econometric evaluation estimator. Review of Economic Studies, 65, 261-294

Hinzmann, S., Cantner, U., \& Graf, H. (2017). The role of geographical proximity for project performance: evidence from the German Leading-Edge Cluster Competition. The Journal of Technology Transfer, doi:10.1007/s10961-017-9600-1.

Lechner, M. (1998). Training the east German labor force: Microeconometric evaluations of continuous vocational training after unification. Heidelberg: Physica Verlag.

Lechner, M. (2001). Identification and estimation of causal effects of multiple treatments under the conditional independence assumption. In M. Lechner \& F. Pfeiffer (Eds.), Econometric evaluation of labor market policies (pp. 43-58). Heidelberg: Physica/Springer.

Leuven, E., \& Sianesi, B. (2003). PSMATCH2: Stata module to perform full Mahalanobis and propensity score matching, common support graphing, and covariate imbalance testing. Statistical Software Components, Boston College Department of Economics. This version: 3.1.4 17dec2008.

Lundvall, B., (1988). Innovation as an interactive process: From user-producer interaction to the national systems of innovation, in: Dosi, G.; Freeman, C.; Nelson, R.; Silverberg, G. \& Soete, L. (Eds.). Technical change and Economic Theory. Pinter, London, 349-369.

Martin, R., Sunley, P., (2003). Deconstructing clusters: chaotic concept or policy panacea?. Journal of Economic Geography, 3(1), 5-35.

Martin, P., Mayer, T., \& Mayneris, F. (2011). Public support to clusters. A firm level study of French local productive systems. Regional Science and Urban Economics, 41, 108-123.

Moodysson, J., Zukauskaite, E., (2014). Institutional Conditions and Innovation Systems: On the Impact of Regional Policy on Firms in Different Sectors. Regional Studies, 48(1), 127-138.

Porter, M.E., (1998). Clusters and the new economics of competition, Harvard Business Review, 76(6), 77-90.

Rothgang, M., Dehio, J., Lageman, B. (2017). Analysing the effects of cluster policy: What can we learn from the German leading-edge cluster competition? The Journal of Technology Transfer, doi:10.1007/s10961-017-9616-6.

Smith, J., \& Todd, P. (2005). Does matching overcome LaLonde's critique of nonexperimental estimators? Journal of Econometrics, 125(1-2), 305-353.

Wallsten, S. J. (2000). The Small Business Innovation Research program: Encouraging technological innovation and commercialization in small firms? RAND Journal of Economics, 31, 82-100.

\section{Submit your manuscript to a SpringerOpen ${ }^{\circ}$ journal and benefit from:}

- Convenient online submission

- Rigorous peer review

- Open access: articles freely available online

- High visibility within the field

- Retaining the copyright to your article

Submit your next manuscript at $>$ springeropen.com 